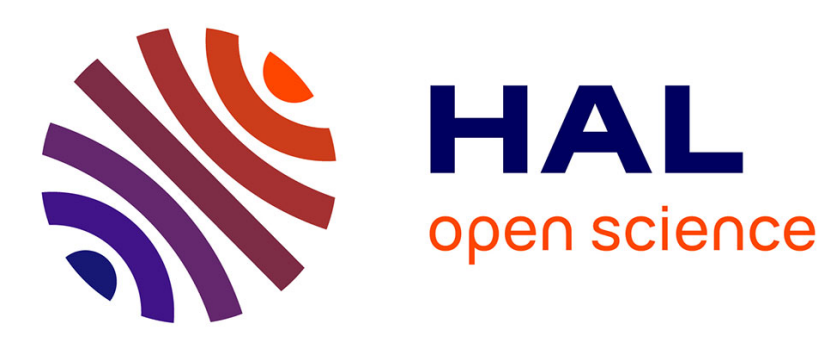

\title{
Analysis and Design of Equilibrium Points for the Cell-Transmission Traffic Model
}

Dominik Pisarski, Carlos Canudas de Wit

\section{To cite this version:}

Dominik Pisarski, Carlos Canudas de Wit. Analysis and Design of Equilibrium Points for the CellTransmission Traffic Model. ACC 2012 - American Control Conference, Jun 2012, Montreal, Canada. hal-00691522v3

\section{HAL Id: hal-00691522 \\ https://hal.inria.fr/hal-00691522v3}

Submitted on 30 Jun 2012

HAL is a multi-disciplinary open access archive for the deposit and dissemination of scientific research documents, whether they are published or not. The documents may come from teaching and research institutions in France or abroad, or from public or private research centers.
L'archive ouverte pluridisciplinaire HAL, est destinée au dépôt et à la diffusion de documents scientifiques de niveau recherche, publiés ou non, émanant des établissements d'enseignement et de recherche français ou étrangers, des laboratoires publics ou privés. 


\title{
Analysis and Design of Equilibrium Points for the Cell-Transmission Traffic Model
}

\author{
Dominik Pisarski and Carlos Canudas-de-Wit
}

\begin{abstract}
The problem of equilibrium points for the Cell Transmission Model is studied. The structure of equilibrium sets is analyzed in terms of model parameters and boundary conditions. The goal is to determine constant input flows, so that the resultant steady state of vehicle density is uniformly distributed along a freeway. The necessary and sufficient conditions for the existence of one-to-one relation between input flow and density are derived. The equilibrium sets are described by formulas that allow to design a desired balanced density. A numerical example for the case of a two-cell system is presented.
\end{abstract}

\section{INTRODUCTION}

Mobility plays an important role in economy as well as in quality of human living. Every day the global impact of well organized transportation is counted in millions of tons fuel saved, thousands of fewer traffic accidents and thousands of kilograms carbon dioxide less emitted. These facts motivate even more intensive research on modeling and control of freeway systems. A good number of different control techniques, based on ramp metering and variable speed limiting, has been developed and put into practice. Most of these techniques enhance the exploitation of traffic systems by optimizing suitable metrics.

The most common metrics in use is the Total Travel Spent (TTS) and the Total Travel Distance (TTD). The former is to decrease the time of travel incurred by all drivers while the latter is to increase the usage of a freeway. TTS and TTD are often mixed with some extra terms, that penalize abrupt variations in ramp metering and speed limiting signals [1].

Most of the gradient optimization methods lead to openloop solutions [2], [3], [4]. The Model Predictive Control procedure to coordinate both variable speed limits and ramp metering was presented in [5]. Optimization procedure based on the Powell's method was used to tune a close-loop regulator in [6]. A close-loop control system based on neural network was proposed in [7]. The sliding mode control method to prevent the propagation of shock waves was presented in [8]. The authors elaborated a decentralized scheme that exploits neighboring cellular information. ALINEA [9], one of the most common in use freeway regulators, optimizes the traffic flow by using a local control law. Some other optimization techniques for freeway systems can be found in [10], [11].

Dominik Pisarski is with NeCS team INRIA, Grenoble, France, dominik.pisarski@inria.fr

C. Canudas-de-Wit is a director of reearch at the CNRS, GIPSA-Lab. $\mathrm{NeCS}$ team, Grenoble, France, carlos.canudas-de-wit@gipsa-lab.grenobleinp. fr
In this paper, we consider the problem of state balancing for freeway traffic system. The state of such a system is represented by distribution of vehicle density while on-ramp demands are assumed for the inputs. The objective is to find input flows that result in a uniform distribution of density. In practice, this uniform distribution can be understood as an equal interdistance between vehicles. The equal interdistance can be attractive to driver's point of view. It reduces the number and intensity of acceleration and deceleration events and therefore, it makes a travel more safety and comfortable, while decreasing emissions.

The problem of state balancing is also studied for other applications. For example, in the paper [12] the authors address the problem of averaging of a stored resource in batteries. They propose a distributed algorithm for flow control. The algorithm guarantees asymptotic convergence of states to the same value that is equal to the average of initial values.

In this paper, we investigate equilibrium sets for freeway system represented by the Cell Transmission Model (CTM). The goal for analysis is to explore balanced equilibria. The results will be crucial for future design of ramp metering strategy. A wide analysis of a behaviour of CTM was previously presented in [13]. The authors proved, that for CTM, every trajectory converges to some equilibrium state. They also showed, that for some cases, a unique input flow may produce an infinite set of equilibrium densities. The following questions then arise. What condition can assure the uniqueness of equilibrium states? What is the input that guarantees the balanced equilibrium state?

The contribution of this paper is the analysis of equilibrium sets for CTM in the context of design of balanced density equilibria. Both the qualitative and quantitative analysis is provided. In particular, the necessary and sufficient conditions for the existence of balanced equilibria are formulated. For the sake of steady state design, a relevant description of the equilibrium sets is given. Results are illustrated in a numerical example.

The organization of the paper is as follows. In the Section II, we give a brief description of CTM, together with its representation as a switched system. The preliminaries and definitions are introduced in the Section III. The Section IV is to analyse the structure of equilibrium sets. In the Section $\mathrm{V}$ we address the problem of design of balanced equilibrium density. Finally, conclusions are given. 


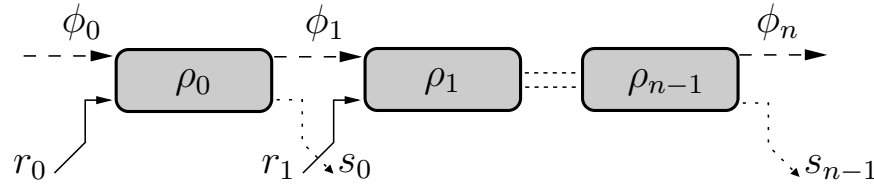

Fig. 1. Freeway divided into $n$ cells. Each cell is accompanied with one on-ramp and one off-ramp.

\section{THE CELL TRANSMISSION MODEL AND STATE SPACE REPRESENTATION}

In this paper, we consider a section of a freeway divided into cells as depicted in Fig. 1. Each cell is assumed to be equipped with one on-ramp and one off-ramp. For such a system, we adopt Daganzo's Cell Transmission Model (CTM), introduced in [14].

For the cell $i$ we assume the following notation: $\rho_{i}$-vehicle density, $\phi_{i}$-mainstream flow entering the cell, $r_{i}$-on-ramp flow entering the cell, $s_{i}$-off-ramp flow leaving the cell, $v_{i}-$ free flow speed, $w_{i}$-congestion wave speed, $F_{i}$-capacity, $\bar{\rho}_{i}-$ jam density, $l_{i}$-cell length. The relation between flow and density is represented by the triangular fundamental diagram (see Fig. 2).

CTM describes the evolution of the system in discrete time $t_{k}=k \Delta t$, with $k \in \mathbb{N}$. Time step $\Delta t>0$ is taken small enough to provide convergence condition (for details see [15]). For the cell $i$ the governing equation is:

$$
\rho_{i}(k+1)=\rho_{i}(k)+\frac{\Delta t}{l_{i}}\left[\Phi_{i}^{+}(k)-\Phi_{i}^{-}(k)\right] .
$$

Here $\Phi_{i}^{+}(k)$ and $\Phi_{i}^{-}(k)$ are total flows entering and leaving the cell $i$, i.e.:

$$
\begin{aligned}
& \Phi_{i}^{+}(k)=\phi_{i}(k)+r_{i}(k), \\
& \Phi_{i}^{-}(k)=\phi_{i+1}(k)+s_{i}(k) .
\end{aligned}
$$

By introducing Demand $D_{i}$ and Supply $S_{i}$ functions:

$$
\begin{aligned}
& D_{i}(k)=\min \left\{\bar{\beta}_{i} v_{i} \rho_{i}(k), F_{i}\right\}, \\
& S_{i}(k)=\min \left\{w_{i}\left(\bar{\rho}_{i}-\rho_{i}(k)\right), F_{i}\right\}
\end{aligned}
$$

according to Daganzo's merge connection model mainstream and on-ramp flows for $i=0,1, \ldots, n-1$ are given as follows:

$$
\begin{aligned}
& \text { if } D_{i-1}(k)+u_{i}(k) \leq S_{i}(k): \\
& \phi_{i}(k)=D_{i-1}(k), r_{i}(k)=u_{i}(k) \\
& \text { otherwise : } \\
& \phi_{i}(k)=\operatorname{mid}\left\{D_{i-1}(k), S_{i}(k)-u_{i}(k),\left(1-p_{i}\right) S_{i}(k)\right\}, \\
& r_{i}(k)=\operatorname{mid}\left\{u_{i}(k), S_{i}(k)-D_{i-1}(k), p_{i} S_{i}(k)\right\} .
\end{aligned}
$$

Here the function mid $\{\cdot\}$ returns the middle value. We will say, that the cell $i$ is in free state if $\rho_{i} v_{i} \leq F_{i}$. Otherwise, it is in congested state.

In (4) we introduced on-ramp demands $u_{i}$ that will appear later as the inputs for the control system. By $p_{i} \in[0,1]$ we denote a parameter, that captures the priorities between mainstream flow $\phi_{i}$ and on-ramp flow $r_{i}$ when merging in a cell during the congestion. In order to determine the value

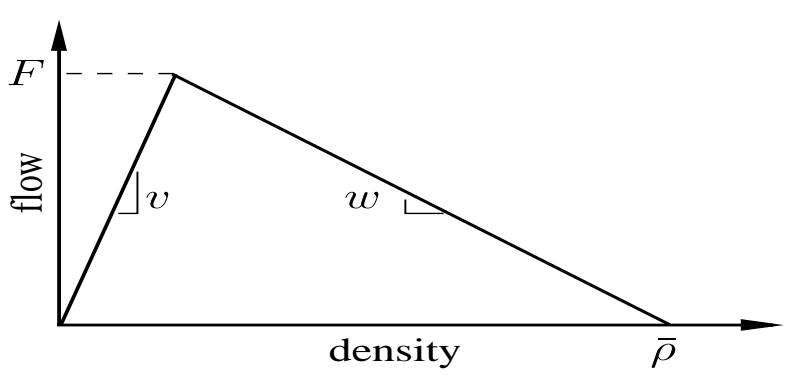

Fig. 2. The fundamental diagram. The shape is determined by the parameters: $F$ - capacity, $v$ - free flow velocity, $w$ - congestion wave speed.

for $p_{i}$ we should consider geometrical properties of on-ramp as well as drivers' behaviour. For the most of the cases, we can assume, that the priority is for the mainstream flow, and therefore we set $p_{i}<0.5$.

The boundary conditions are determined by some demand function $\bar{D}(k)=D_{-1}(k)$ for the cell 0 and some supply function $\bar{S}(k)=S_{n}(k)$ for the cell $n-1$. For the flow $\phi_{n}$ we adopt the simple connection model, and therefore:

$$
\phi_{n}(k)=\min \left\{D_{n-1}(k), \bar{S}(k)\right\} .
$$

Finally, for the off-ramp flows we assume:

$$
s_{i}(k)=\frac{1-\bar{\beta}_{i}}{\bar{\beta}_{i}} \phi_{i+1}(k) .
$$

Here $\bar{\beta}_{i}=1-\beta_{i}$, where $\beta_{i} \in[0,1)$ stands for the split ratio, which is the parameter, that specifies a number of vehicles leaving the cell $i$.

For the convenience of our further investigation, we rewrite the governing equation (1) in a form of a switched control system:

$$
\begin{aligned}
& x(k+1)=A_{s(k)} x(k)+B_{s(k)} u(k)+C_{s(k)}(k), \\
& s(k)=f(x(k), u(k)) .
\end{aligned}
$$

Here the state vector $x \in \mathcal{X}$ and the input vector $u \in \mathcal{U}$ stand for vehicle densities and input flows (on-ramp demands), respectively:

$$
x=\left(\rho_{0}, \rho_{1}, \ldots, \rho_{n-1}\right), u=\left(u_{0}, u_{1}, \ldots, u_{n-1}\right) .
$$

The sets $\mathcal{X} \in \mathcal{R}^{n}$ and $\mathcal{U} \in \mathcal{R}^{n}$ respect minimum and maximum values for densities and on-ramp demands. The switching variable $s \in \mathcal{S}=\{1,2, \ldots, m\}$ indicates a mode of the system, and it determines terms in the matrices $A^{n \times n}$, $B^{n \times n}$ and for the vector $C^{n \times 1}(k)$. The vector $C^{n \times 1}(k)$ consist of the boundary functions $\bar{D}(k)$ and $\bar{S}(k)$. Notice that $m$ is a total number of all possible modes generated by nonlinear formulas for mainstream and on-ramp flows.

\section{PROBLEM FORMULATION}

As it was stated in the introduction, the goal of this paper is to analyse the structure of equilibrium sets for CTM. In particular, we will investigate sets of balanced equilibrium points. Before going into the analysis, we first introduce some formal definitions. 
Definition The equilibrium set $\Delta$ for CTM described by (7) is a set of pairs $\left(x^{*}, u^{*}\right)$ that under a fixed boundary conditions solve the following equilibrium equation:

$$
\begin{aligned}
& \left(A_{s}-\mathbf{I}\right) x^{*}+B_{s} u^{*}+C_{s}=0, \\
& s=f\left(x^{*}, u^{*}\right), \quad s \in \mathcal{S} .
\end{aligned}
$$

Here I stands for the identity matrix. In terms of (1) the steady state is reached when for every cell, the total flow entering and leaving are equal. Notice that vectors $C_{s}$ becomes constant, when boundary conditions have fixed value, i.e.: $\bar{D}(k)=\bar{D}$ and $\bar{S}(k)=\bar{S}$. By $\Delta_{x}$ and $\Delta_{u}$ we denote the set of equilibrium density and equilibrium input flow, respectively.

In order to analyse the structures of $\Delta$ we need to represent it in a more convenient way. This can be done by introducing a relevant set of inequalities. First, we decompose $\Delta$ into subsets that correspond to the modes of the system. For every $s \in \mathcal{S}$ we define the following set:

$$
\begin{aligned}
\Delta_{s}=\left\{\left(x^{*}, u^{*}\right):\right. & \left(A_{s}-\mathbf{I}\right) x^{*}+B_{s} u^{*}+C_{s}=0, \\
& \left.s=f\left(x^{*}, u^{*}\right)\right\} .
\end{aligned}
$$

Then we have $\Delta=\bigcup_{s=1}^{m} \Delta_{s}$. The function $f$ results from $\operatorname{mid}\{\cdot\}$ and $\min \{\cdot\}$ terms in (3)-(5). Therefore, by introducing relevant inequalities we can rewrite every $\Delta_{s}$ as:

$$
\begin{aligned}
\Delta_{s}=\left\{\left(x^{*}, u^{*}\right):\right. & \left(A_{s}-\mathbf{I}\right) x^{*}+B_{s} u^{*}+C_{s}=0, \\
& \left.K_{s} x^{*}+L_{s} u^{*}+M_{s} \leq 0\right\} .
\end{aligned}
$$

Here the matrices $K_{s}, L_{s}$ and the vector $M_{s}$ are constant. To illustrate how to build inequalities in (11) we give an example for the case of a two-cell system. Using (1)-(6) we can write:

$$
\begin{aligned}
& \text { for } s=1: \bar{D}+u_{0}^{*}=\frac{1}{\bar{\beta}_{0}} D_{0}, D_{0}+u_{1}^{*}=\frac{1}{\bar{\beta}_{1}} D_{1}, \\
& \bar{D}+u_{0}^{*} \leq S_{0}, D_{0}+u_{1}^{*} \leq S_{1}, D_{1} \leq \bar{S}, \\
& \text { for } s=2: \quad S_{0}=\frac{1}{\bar{\beta}_{0}} D_{0}, D_{0}+u_{1}^{*}=\frac{1}{\bar{\beta}_{1}} D_{1}, \\
& \bar{D}+u_{0}^{*} \geq S_{0}, D_{0}+u_{1}^{*} \leq S_{1}, D_{1} \leq \bar{S},
\end{aligned}
$$

and so forth, till $s=m$.

Inserting relevant formulas for Demand and Supply functions for $s=1$ we have:

$$
\begin{aligned}
& {\left[\begin{array}{cc}
-v_{0} & 0 \\
\bar{\beta}_{0} v_{0} & -v_{1}
\end{array}\right]\left[\begin{array}{l}
x_{0}^{*} \\
x_{1}^{*}
\end{array}\right]+\left[\begin{array}{ll}
1 & 0 \\
0 & 1
\end{array}\right]\left[\begin{array}{l}
u_{0}^{*} \\
u_{1}^{*}
\end{array}\right]+\left[\begin{array}{c}
\bar{D} \\
0
\end{array}\right]=0,} \\
& {\left[\begin{array}{cc}
w_{0} & 0 \\
\bar{\beta}_{0} v_{0} & w_{1} \\
0 & \bar{\beta}_{1} v_{1}
\end{array}\right]\left[\begin{array}{l}
x_{0}^{*} \\
x_{1}^{*}
\end{array}\right]+\left[\begin{array}{ll}
1 & 0 \\
0 & 1 \\
0 & 0
\end{array}\right]\left[\begin{array}{l}
u_{0}^{*} \\
u_{1}^{*}
\end{array}\right]+\left[\begin{array}{c}
\bar{D}-w_{0} \bar{\rho}_{0} \\
-w_{1} \bar{\rho}_{1} \\
-\bar{S}
\end{array}\right] \leq 0 .}
\end{aligned}
$$

For the set $\Delta$ we will pay the special attention to these points $\left(x^{*}, u^{*}\right)$, where $x^{*}$ are balanced. Before we describe this balance, let us introduce a set of admissible balanced density:

$$
\mathcal{C}=\left\{c: 0 \leq c \leq \min \left\{\bar{\rho}_{i}\right\}_{i=0}^{n-1}\right\}
$$

Then the set of balanced equilibrium points $\Delta_{B} \subset \Delta$ is defined as follows:

Definition $\Delta_{B}$ is a set of equilibrium points $\left(x^{*}, u^{*}\right)$, such that the equilibrium density is balanced, i.e.:

$$
x^{*}=c \mathbf{1}, \quad c \in \mathcal{C} .
$$

Here $1^{n \times 1}$ stands for all-ones vector. By analogy to the set $\Delta$ we will denote by $\Delta_{x B}$ and $\Delta_{u B}$ the set of balanced equilibrium densities and the set of related equilibrium input flows, respectively.

The objectives of this paper are as follows. To determine how the shape of $\Delta$ corresponds to model parameters. To derive a condition, that ensures the existence of the set $\Delta_{B}$. To provide formulas, that enable us to find input flows $u^{*}$, so that the resulting equilibrium density $x^{*}$ is balanced.

Before we go further, we need to recall some basic facts on the relation between the sets $\Delta_{u}$ and $\Delta_{x}$. In the paper [13], it was presented, that in general the mapping $\Delta_{u} \longrightarrow \Delta_{x}$ is not one-to-one. The authors provided a simple example, where they fixed values for demands and input flows. The resulting equilibrium density was not a unique point. It was represented by some set of points instead. It was shown, that the steady state was determined not only by the input but also by the initial state. Therefore, for such a case, the design of density equilibria is not feasible. In this paper, we consider only these cases for which any fixed input flow results in a unique equilibrium density. Moreover, we focus only on these points of $\left(x^{*}, u^{*}\right) \in \Delta$ such that the mapping $\Delta_{u} \longrightarrow \Delta_{x}$ is bijective. Notice that $\Delta_{u} \longrightarrow \Delta_{x}$ is bijective for the mode $s$ iff both $\left(A_{s}-\mathbf{I}\right)$ and $B_{s}$ are invertible.

\section{STRUCTURE OF EQUILIBRIUM SETS}

In this section, we describe the structure of the set $\Delta$. As stated before, we limit our investigation to these equilibria that respect the one-to-one property. From (4) we can observe that matrix $B_{s}$ is invertible iff the following conditions are satisfied:

$$
\begin{aligned}
& \bar{D}+u_{0}^{*} \leq S_{0}, \\
& D_{i-1}+u_{i}^{*} \leq S_{i}, \quad i=1,2, \ldots, n-1 .
\end{aligned}
$$

Under this assumption, the equilibrium equation in (9) is represented by:

$$
\begin{aligned}
& \bar{D}+u_{0}^{*}=\frac{1}{\bar{\beta}_{0}} D_{0}, \\
& D_{i-1}+u_{i}^{*}=\frac{1}{\bar{\beta}_{i}} D_{i}, \quad i=1,2, \ldots, n-2, \\
& D_{n-2}+u_{n-1}^{*}=\frac{1}{\bar{\beta}_{n-1}} \min \left\{D_{n-1}, \bar{S}\right\}
\end{aligned}
$$

To ensure the invertibility of $\left(A_{s}-\mathbf{I}\right)$ we need to impose additional conditions, i.e.:

$$
D_{n-1} \leq \bar{S}, \quad \bar{\beta}_{i} v_{i} x_{i}^{*} \leq F_{i}, \quad i=0,1, \ldots, n-1 .
$$

Under the condition (18) we can rewrite (17) as follows:

$$
\begin{aligned}
& \bar{D}+u_{0}^{*}=v_{0} x_{0}^{*}, \\
& \bar{\beta}_{i-1} v_{i-1} x_{i-1}^{*}+u_{i}^{*}=v_{i} x_{i}^{*}, \quad i=1,2, \ldots, n-1 .
\end{aligned}
$$


Let us define $\Gamma$ as the one-to-one mapping $\Delta_{u} \longrightarrow \Delta_{x}$ described by (19).

Based on (16)-(18) we can formulate the necessary condition for the existence of $\Gamma$ in terms of boundary and input flows.

Proposition 4.1: There exist the mapping $\Gamma$ only if :

$$
\prod_{i=0}^{n-1} \bar{\beta}_{i} \bar{D}+\sum_{i=0}^{n-1}\left(\prod_{j=i}^{n-1} \bar{\beta}_{j} u_{i}^{*}\right) \leq \bar{S}
$$

Proof: From (18) we have:

$$
D_{n-1} \leq \bar{S}
$$

Inserting $D_{n-1}$ from (17) we obtain:

$$
\bar{\beta}_{n-1} D_{n-2}+\bar{\beta}_{n-1} u_{n-1}^{*} \leq \bar{S} .
$$

Repeating this substitution from $D_{n-2}$ to $D_{0}$ we get (20).

For the analysis of the equilibrium set we consider only two special cases. In the first case we assume, that all cells are in the free state. Then we describe the shape of $\Delta$ in terms of system parameters. The second case is to show, that for the congested state, the conditions (16)-(18) drive to contradiction.

\section{A. Set of free equilibrium points}

Definition The set of free equilibrium points $\Delta^{f} \subset \Delta$ is a set of pairs $\left(x^{*}, u^{*}\right)$ where $x^{*}$ is the free state, i.e.:

$$
v_{i} x_{i}^{*} \leq F_{i}, \quad i=0,1, \ldots, n-1 .
$$

Consistently, we will denote by $\Delta_{x}^{f}$ and $\Delta_{u}^{f}$ the sets of free equilibrium densities and sets of corresponding equilibrium input flows, respectively

Now we will formulate the sufficient condition for the existence of $\Gamma$ under $x^{*} \in \Delta_{x}^{f}$. First we show, that the condition:

$$
\bar{\beta}_{i} v_{i} x_{i}^{*} \leq F_{i}, \quad i=0,1, \ldots, n-1 .
$$

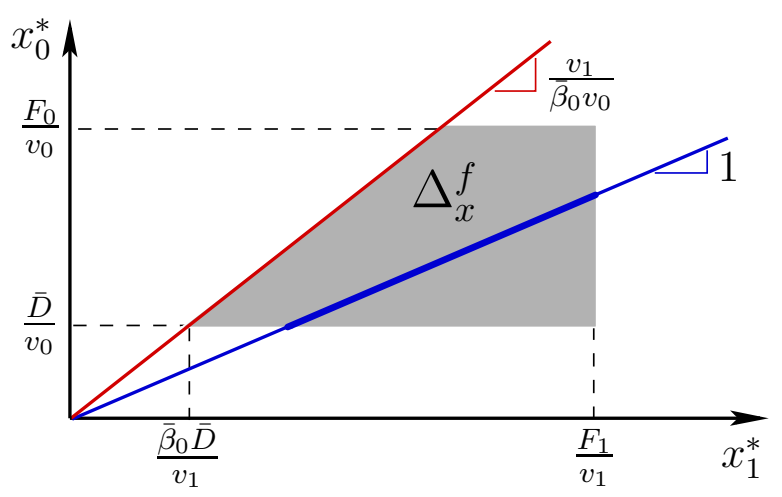

Fig. 3. An example of the set of free equilibrium densities for the case of two-cell system. The grey trapezium is for the set $\Delta_{x}^{f}$. The blue line indicates balanced states.

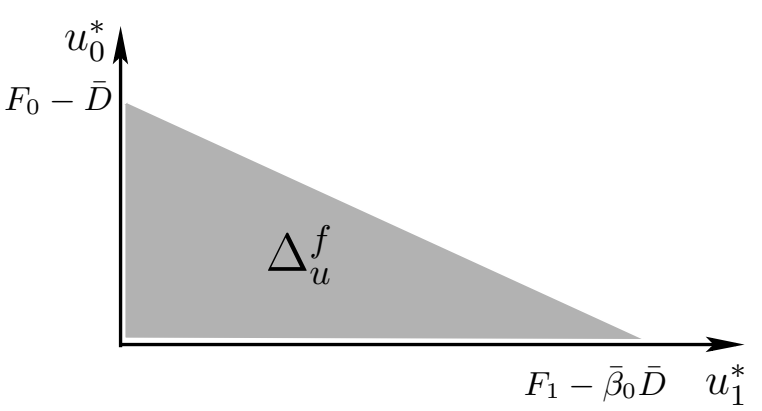

Fig. 4. An example of the set of free equilibrium input flows for the case of two-cell system. The grey triangle is for the set $\Delta_{u}^{f}$.

is satisfied. Indeed, it is because for $\bar{\beta}_{i} \leq 1$ we have:

$$
\bar{\beta}_{i} v_{i} x_{i}^{*} \leq v_{i} x_{i}^{*} \leq F_{i}, \quad i=0,1, \ldots, n-1 .
$$

Proposition 4.2: Let $x^{*}$ be some free state equilibrium. Then, there exist the mapping $\Gamma$ if the following conditions hold:

$$
\begin{aligned}
& \bar{D}+u_{0}^{*} \leq F_{0} \\
& \bar{\beta}_{i-1} v_{i-1} x_{i-1}^{*}+u_{i}^{*} \leq F_{i}, \quad i=1,2, \ldots, n-1, \\
& \bar{\beta}_{n-1} v_{n-1} x_{n-1}^{*} \leq \bar{S}
\end{aligned}
$$

Finally, we can describe the sets $\Delta_{x}^{f}$ and $\Delta_{u}^{f}$ as follows:

$$
\begin{aligned}
\Delta_{x}^{f}=\{ & x^{*} \in \mathcal{X}: \\
& v_{i} x_{i}^{*} \leq F_{i}, i=0,1, \ldots, n-1, \\
& x_{0}^{*}=\frac{\bar{D}+u_{0}^{*}}{v_{0}} \\
& x_{i}^{*}=\frac{\bar{\beta}_{i-1} v_{i-1} x_{i-1}^{*}+u_{i}^{*}}{v_{i}}, i=1,2, \ldots, n-1, \\
& \left.u^{*} \in \Delta_{u}^{f}\right\}, \\
\Delta_{u}^{f}=\{ & u^{*} \in \mathcal{U}: \\
& u_{0}^{*} \leq F_{0}-\bar{D}_{1} \\
& u_{1}^{*} \leq F_{1}-\bar{\beta}_{0}\left(\bar{D}_{1}+u_{0}^{*}\right), \\
& u_{2}^{*} \leq F_{2}-\bar{\beta}_{1}\left(\bar{\beta}_{0}\left(\phi_{0}+u_{0}^{*}\right)+u_{1}^{*}\right) \\
& \text { and so forth }\} .
\end{aligned}
$$

In Fig. 3 we present the shape of $\Delta_{x}^{f}$ (grey trapezium) for the case of a two-cell system. The corresponding set $\Delta_{u}^{f}$ is represented by grey triangle depicted in Fig. 4. We can observe how the system parameters affect on $\Delta_{x}^{f}$. For instance, if the slope of the left bound (indicated by red line) is less then 1 , then the set is represented by a triangle placed below the blue line indicating balanced states. Therefore, in this case the set $\Delta_{x B}$ would be empty. We will explore this fact in details in the section $\mathrm{V}$.

\section{B. Set of congested equilibrium points}

Definition The set of congested equilibrium points $\Delta^{c} \subset \Delta$ is a set of pairs $\left(x^{*}, u^{*}\right)$ where $x^{*}$ is the congested state, i.e.:

$$
v_{i} x_{i}^{*}>F_{i}, i=0,1, \ldots, n-1 .
$$


Now we will show, that under the conditions (16)-(18) the set $\Delta^{c}$ is empty. For the congested case $\left(S_{i}<F_{i}\right.$ for all $\left.i\right)$ the condition in (16) is as follows:

$$
\bar{D}+u_{0} \leq S_{0}<F_{0} .
$$

On the other we have assumed that:

$$
x_{0}^{*} v_{0}>F_{0} \text {. }
$$

Thus the equality:

$$
\bar{D}+u_{0}=x_{0}^{*} v_{0}
$$

is a contradiction and therefore, for the congested case the mapping $\Gamma$ does not exist.

\section{DESIGN OF BALANCED EQUILIBRIUM STATES}

So far, we have derived the necessary and sufficient conditions for the existence of $\Gamma$ - the relation, that allows as to design equilibrium densities $x^{*} \in \Delta_{x}$. In order to design balanced equilibrium $x^{*} \in \Delta_{x B}$, we need to impose some additional requirements.

In the previous section, we proved that $\Gamma$ exists only for the free state case. Therefore, for the admissible balanced densities we consider only these points $c \in \mathcal{C}$ that satisfy the condition:

$$
c \leq \min \left\{\frac{F_{i}}{v_{i}}\right\}_{i=0}^{n-1} .
$$

Then the set of balanced equilibrium points $\Delta_{B}$ can be defined as a set of pairs $\left(u^{*}, c\right)$, that solve the equations:

$$
\begin{aligned}
& \bar{D}+u_{0}^{*}=v_{0} c, \\
& \bar{\beta}_{i-1} v_{i-1} c+u_{i}^{*}=v_{i} c, i=1,2, \ldots, n-1 .
\end{aligned}
$$

Respecting real freeway scenarios we have to assume:

$$
u^{*} \geq 0 \text {. }
$$

Then we can formulate the necessary condition for the existence of balanced equilibria.

Proposition 5.1: Let $u_{i}^{*} \geq 0$ for $i=0,1, \ldots, n-1$. Then, there exists a balanced free equilibrium point only if :

$$
\bar{\beta}_{i-1} v_{i-1} \leq v_{i}, \quad i=1,2, \ldots, n-1 .
$$

Proof: Let us assume $\bar{\beta}_{i-1} v_{i-1}>v_{i}$ for $i=$ $1,2, \ldots, n-1$. Then (34) is a contradiction under $u \geq 0$.

Remark A practical fact comes up with the Proposition 5.1. In order to design a balanced equilibrium point we may need to apply the variable speed limiting, where the speed limits depend on actual values of the split ratios.

Finally, we can describe the sets of balanced equilibrium points:

$$
\begin{aligned}
\Delta_{x B}^{f}=\left\{x^{*}\right. & =c \mathbf{1}: \\
c & \leq \min \left\{\frac{F_{i}}{v_{i}}\right\}_{i=0}^{n-1}, c=\frac{\bar{D}+u_{0}^{*}}{v_{0}}, \\
u^{*} & \left.\in \Delta_{u B}^{f}\right\}
\end{aligned}
$$

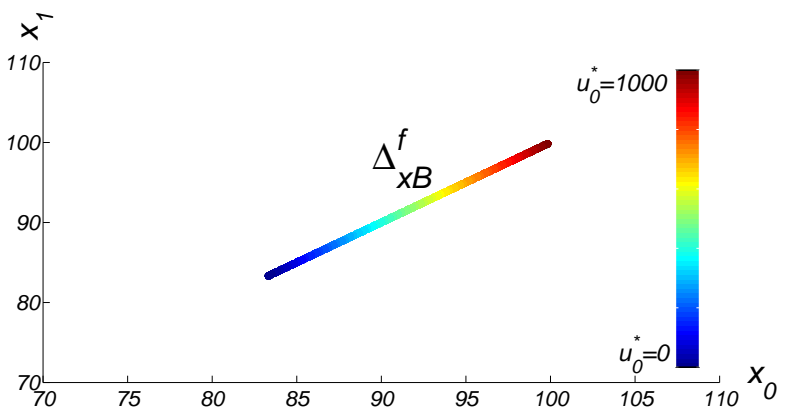

Fig. 5. Numerical example for the set $\Delta_{x B}^{f}$ (color line). The left end of the line corresponds to the input flow vector: $u_{0}^{*}=0, u_{1}^{*}=0$ while the right one is for $u_{0}^{*}=1000, u_{1}^{*}=0$.

$$
\begin{aligned}
\Delta_{u B}^{f}=\{ & u^{*} \in \mathcal{U}: \\
& u_{0}^{*} \leq v_{0} \min \left\{\frac{F_{i}}{v_{i}}\right\}_{i=0}^{n-1}-\bar{D}, \\
& u_{i}^{*} \leq\left(v_{i}-\bar{\beta}_{i-1} v_{i-1}\right) \min \left\{\frac{F_{i}}{v_{i}}\right\}_{i=0}^{n-1}, \\
& \left.\frac{\bar{D}+u_{0}^{*}}{v_{0}}=\frac{u_{i}^{*}}{v_{i}-\bar{\beta}_{i-1} v_{i-1}}, i=1,2, \ldots, n-1\right\},
\end{aligned}
$$

\section{A. Optimal balanced equilibrium point}

Among the points $x^{*} \in \Delta_{x B}^{f}$ we can find the one that optimizes some objective function. For example, we can consider the balanced equilibrium state that maximizes the total input volume (TIV). We define TIV as follows:

$$
J_{T I V}=\sum_{i=0}^{n-1} u_{i}^{*} .
$$

Then the optimal balanced equilibrium density is:

$$
x^{* *}=\frac{\bar{D}+u_{0}^{* *}}{v_{0}} \mathbf{1},
$$

where $u_{0}^{* *}$ solves the following optimization problem:

$$
\begin{aligned}
\text { Find } & u_{0}^{* *}=\max u_{0}^{*}, \\
\text { under } & u^{*} \in \mathcal{U}, \\
& u_{0}^{*} \leq v_{0} \min \left\{\frac{F_{i}}{v_{i}}\right\}_{i=0}^{n-1}-\bar{D}, \\
& u_{i}^{*} \leq\left(v_{i}-\bar{\beta}_{i-1} v_{i-1}\right) \min \left\{\frac{F_{i}}{v_{i}}\right\}_{i=0}^{n-1}, \\
& \frac{\bar{D}+u_{0}^{*}}{v_{0}}\left(v_{i}-\bar{\beta}_{i-1} v_{i-1}\right) \leq u_{i}^{*}, \\
& i=1,2, \ldots, n-1 .
\end{aligned}
$$

\section{B. Numerical example}

To illustrate the results, we present the numerical example for a two-cell system. We assume that all cells have the same free flow speeds and there is no off-ramps $\left(\bar{\beta}_{0}=\bar{\beta}_{1}=1\right)$. In this case, from (34) we immediately conclude, that a 


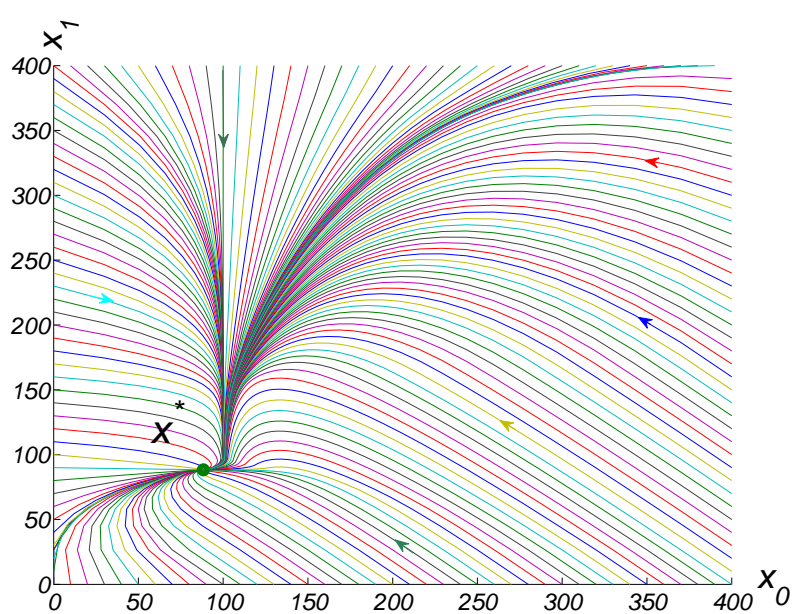

Fig. 6. State orbits (color curves) and balanced equilibrium density (green dot) for the case of $u_{0}^{*}=500, u_{1}^{*}=0$.

balanced equilibrium exists only if the downstream input flow is equal to zero, i.e. $u_{1}^{*}=0$ and only the first on-ramp serves the vehicles $u_{0}^{*} \geq 0$. The parameters are assumed as follows: $v_{0}=v_{1}=60(\mathrm{~km} / \mathrm{h}), w_{0}=w_{1}=20(\mathrm{~km} / \mathrm{h})$, $F_{0}=F_{1}=6000(\mathrm{veh} / \mathrm{h}), \bar{\rho}_{0}=\bar{\rho}_{1}=400(\mathrm{veh} / \mathrm{km})$, $\bar{D}=5000(\mathrm{veh} / \mathrm{h})$. Then the following is the admissible input flow vector: $u_{0}^{*} \in[0,1000](\mathrm{veh} / \mathrm{h})$ and $u_{1}^{*}=0$. The corresponding set $\Delta_{x B}^{f}$ is plotted in Fig 5 as color line. Colors correspond to the values of $u_{0}^{*}$ as indicated on the bar.

In Fig. 6 we present the state trajectories for the case of $u_{0}^{*}=500(\mathrm{veh} / \mathrm{h})$ and $u_{1}^{*}=0$. The attractive point is $x_{0}^{*}=x_{1}^{*}=91.6(\mathrm{veh} / \mathrm{km})$.

\section{CONCLUSIONS AND FUTURE WORKS}

In this paper, the analysis of structure of equilibrium sets for the Cell Transmission Traffic Model has been presented. We have limited our attention to the case, that provides one-to-one mapping between inputs and equilibrium states. This case requires two conditions to be satisfied. First, total demand weighted with split ratios must not exceed supply for the downstream boundary. Second, all cells are in the free state. Under these conditions, we have shown, that the balanced state exists only if the additional relation between free flow velocities and split ratios holds. This suggests that in practical design of balanced equilibria, we may need to use both ramp metering and variable speed limiting, where speed limits depend on actual values of split ratios. For the special case, where parameters for all cells are equal and there is no off-ramps, the balanced state is possible only if the first on-ramp is serving cars while others are closed. In future works, we will address the problem of state balancing for the cases, where the necessary conditions presented here are not fulfilled. The idea is to approach near balanced state while staying within the equilibrium set. This will be formulated as an optimization problem. Further step will be to find near balanced state, so that some objective (for example, TTD) is optimized.

\section{ACKNOWLEDGMENTS}

The research leading to these results has received funding from the European Union Seventh Framework Programme [FP7/2007-2013] under grant agreement $\mathrm{n}^{0} 257462$ HYCON2 Network of excellence.

\section{REFERENCES}

[1] X. Y. Lu, T. Z. Qiu, P. Varaiya, R. Horowitz, S. E. Shladover, Combining Variable Speed Limits with Ramp Metering for Freeway Traffic Control, IEEE American Control Conference, 2010, pp 22662271.

[2] G. Gomes, R. Horowitz, Globally Optimal Solutions to the OnRamp Metering Problem - Part 1, IEEE Conference on Intelligent Transportation Systems, 2004, pp 509-514.

[3] D. Jacquet, C. Canudas-de-Wit, D. Koenig, Optimal Control of Systems of Conservation Laws and Application to Non-Equilibrium Traffic Control, Proceedings of the 13th IFAC Workshop on Control Applications of Optimisation, 2006.

[4] D. Jacquet, C. Canudas-de-Wit, D. Koenig, Optimal Ramp Metering Strategy with Extended LWR Model; Analysis and Computational Methods, 6th IFAC World Congress, 2005.

[5] A. Hegyi, B. De Schutter, H. Hellendroon, T. van den Boom, Optimal Coordination of Ramp Metering and Variable Speed Control - An MPC Approach, IEEE American Control Conference, 2002, pp 36003605 .

[6] A. Alessandri, A. Di Febbraro, A. Ferrara, E. Punta. Optimal Control of Freeways via Speed Signalling and Ramp Metering, Control Engineering Practice, vol. 6, 1998, pp 771-780.

[7] A. Di Febbraro, T. Parisini, S. Sacone, R. Zoppoli, Neural Approximation for Feedback Optimal Control of Freeway Systems, IEEE Transactions on Vehicular Technology, vol. 50(1), 2001, pp 302-312.

[8] Y. H. Chiang, J. Ch. Yuang, Decentralized Control of Freeway Traffic Flow with Neighboring Cellular Information, IEEE International Conference on Systems, Man and Cybernetics, 2006, pp 2547-2552.

[9] M. Papageorgiou, H. Hadj-Salem, J. M. Blosseville, ALINEA: A Local Feedback Control Law for On-ramp Metering, Transportation Research Record, vol. 1320, 1991, pp 58-64.

[10] H.J. Kushner, K. M. Ramachandran, Optimal and Approximately Optimal Control Policies for Queues in Heavy Traffic, SIAM Journal on Control and Optimization, vol. 27, 1989, pp 1293-1318.

[11] M. Gugat, M. Herty, A. Klar, G.Leugering, Optimal Control for Traffic Flow Networks, Journal of Optimization Theory and Applications, vol. 126, 2005, pp 589-616.

[12] M. Baric, F. Borelli, Distributed Averaging with Flow Constraints, IEEE American Control Conference, 2011, pp 4834-4839.

[13] G. Gomes, R. Horowitz, A. A. Kurzhanskiy, P. Varaiya, J. Kwon, Behavior of The Cell Transmission Model and Effectiveness of Ramp Metering, Transportation Research Part C, vol. 16, 2008, pp 485-513.

[14] C. Daganzo, The Cell Transmission Model: A Dynamic Representation of Highway Traffic Consistent with the Hydrodynamic Theory, Transportation Research Part B, vol. 28, 1994, pp 269-287.

[15] R. J. LeVeque, Numerical Methods for Conservation Laws, Birkhauser Verlag, Basel; 1992. 